\title{
根表面养分吸收通量和根围溶质浓度的近似解析解
}

\author{
张慧萍 ${ }^{1}$ 王淑月 ${ }^{1}$ 欧忠辉1,2* \\ ${ }^{1}$ 福建师范大学数学与信息学院, 福州 $350117,{ }^{2}$ 福建省分析数学及其应用重点实验室, 福州 350117
}

摘 要 该文用Nye-Tinker-Barber模型来研究植物根系表面的养分吸收通量和根围溶质浓度的近似解析解。将根围区域分为 远场区域和近场区域, 在远场用相似变量, 在近场用尺度变换, 将远场解在根表面展开并与近场解进行待定函数的匹配, 从 而获得对流扩散方程根表面通量和浓度的一阶近似解析解, 该解能够简化到扩散方程的解的形式。对氮、钾、硫、磷、镁、 钻的养分吸收通量和氮、钾的浓度分别进行数值模拟，比较模型的数值解、Roose的近似解析解和该文的近似解析解。结果 表明: 在扩散方程中, 6种元素通量的解析解与Roose解析解相近, 但均高于数值解, 钾和磷的通量在短时间内迅速衰减; 钾 和氮浓度的全局近似解析解与Roose解析解接近, 并与数值解的变化趋势一致。在对流扩散方程中, 除氮外的 5 种元素通量的 近似解较Roose的解析解更接近于数值解, 且没有奇性。

关键词 解析解; 养分吸收通量; Nye-Tinker-Barber模型; 溶质浓度全局解

张慧萍, 王淑月, 欧忠辉 (2018). 根表面养分吸收通量和根围溶质浓度的近似解析解. 植物生态学报, 42, 1043-1049. DOI: 10.17521/cjpe.2018.0117

\section{Approximate analytical solutions of root surface nutrient uptake flux and rhizosphere solute concentrations}

ZHANG Hui-Ping ${ }^{1}$, WANG Shu-Yue ${ }^{1}$, and OU Zhong-Hui ${ }^{1,2 *}$

${ }^{1}$ College of Mathematics and Informatics, Fujian Normal University, Fuzhou 350117, China; and ${ }^{2}$ Fujian Key Laboratory of Mathematical Analysis and Applications, Fuzhou 350117, China.

\section{Abstract}

Aims We use the Nye-Tinker-Barber model to obtain approximate analytical solutions of the root surface nutrient uptake flux and the rhizosphere solute concentrations.

Methods The rhizosphere is divided into the distant field and the close-range field. The outer solution is obtained by the similarity variable method, and the inner solution is obtained by the rescaling method. Expanding the outer solution from root surface and matching it with the inner solution, we finally obtain the approximate analytical solutions of the root surface nutrient uptake flux and rhizosphere solute concentrations based on Nye-Tinker-Barber model. Numerical simulations are performed on the uptake fluxes of six elements (N, K, P, $\mathrm{Mg}, \mathrm{S}$ and $\mathrm{Ca}$ ) and the solute concentrations of two elements ( $\mathrm{N}$ and $\mathrm{K}$ ). Comparisons are made among the NyeTinker-Barber model approximate analytical solutions, Roose's analytical solutions and the numerical solutions.

Important findings The approximate analytical solutions of the root surface uptake fluxes based on the diffusion Nye-Tinker-Barber model are similar to those of the Roose's analytical solutions; both approaches produced higher values than the numerical solutions. The approximate analytical solutions of the rhizosphere $\mathrm{N}$ and $\mathrm{K}$ concentrations based on the diffusion Nye-Tinker-Barber model are similar to the Roose's analytical solutions and coincide with the trend of changes in the numerical solutions. The approximate analytical solutions of all elements but $\mathrm{N}$, of the root surface, uptake fluxes based on the convection-diffusion Nye-Tinker-Barber model are more similar to the numerical solutions than the Roose's analytical solutions, and the approximate analytical solutions of the rhizosphere $\mathrm{N}$ and $\mathrm{K}$ concentrations are coincide with the trend of changes in the numerical solutions.

Key words analytical solution; nutrient absorption flux; the Nye-Tinker-Barber model; global solution of solute concentration

Zhang HP, Wang SY, Ou ZH (2018). Approximate analytical solutions of root surface nutrient uptake flux and rhizosphere solute concentrations. Chinese Journal of Plant Ecology, 42, 1043-1049. DOI: 10.17521/cjpe.20188.0117

收稿日期Received: 2018-05-17 接受日期Accepted: 2018-08-23

基金项目：国家自然科学基金(11671085和11771082)和福建省引导性项目(2015Y0054)。Supported by the National Natural Science Foundation of China

(11671085 and 11771082), and the Fujian Province Guiding Project (2015Y0054).

* 通信作者Corresponding author (zhou@fjnu.edu.cn) 
植物根系具有固定植物、吸收养分、储存水分、 合成运输有机物等众多重要功能(陈红等, 2013)。目 前已知高等植物需要的16-18种营养元素除碳、氢、 氧可以从大气中获取外, 其余如氮、磷、钾、铝、 镁、硫、铁、锰、锌、铜等都必须依靠根系从土壤 中汲取。养分一般以截取、离子扩散、质流这三种 方式向根表面迁移, 但由于土壤颗粒与根的接触面 积有限, 因此截取量很少(仅为植物需要量的 $5 \%$ ), 远远不能满足植物的生长需要, 还必须通过扩散和 质流获取更多的养分(蒙好生等, 2017)。早在20世纪 60 年代植物学家就开始了对根部吸收养分过程的研 究。Bouldin (1961)假设根的养分吸收通量与养分浓 度成正比关系, 建立了土壤养分扩散模型。随后 Olsen等(1962)通过假设恒定的养分吸收通量或者养 分浓度, 建立了类似的模型, 但是这类模型只适用 于像磷和钾这样以扩散为主要的迁移方式的养分离 子, 不适合像氮、硫、钠等以对流为主的养分离子。

Nye和Spiers (1964)建立了一个同时考虑对流 和扩散的模型。因为养分和水分向根表面移动的动 力是根表面对养分吸收后形成的浓度差, 所以 Epstein (1972)提出用米氏动力学方程作为养分吸收 模型的边界条件来描述根表面的养分吸收, 该边界 条件是非线性的。Nye, Tinker和Barber等研究者, 在 Nye和Spiers的模型基础上, 同时考虑对流和扩散过 程, 建立了大范围内养分向根部迁移的圆柱形单根 养分吸收模型, 简称Nye-Tinker-Barber模型(Nye \& Tinker, 1977; Barber, 1984)。在此模型的基础上使用 平均化方法修正根对土壤养分扩散吸收或者在对流 扩散方程中增加源汇项。Roose和Fowler (2004)建立 了植物根系同时吸收水分和养分的模型; Schnepf和 Roose (2006)建立了菌丝体养分吸收模型; Schnepf 等(2011)建立了单个菌丝养分吸收模型; Zygalakis 等(2011)建立了根毛的养分吸收的双重孔隙模型; Zygalakis和Roose (2012)建立了主根和排根分泌柠 檬酸盐对根系吸收养分影响的模型; Badge和 Adlakha (2017)建立了水通量对玉米根基硝酸盐动 态影响的二维有限元模型; Kumar和Hafiz (2011)用 此模型研究植物根部的污染物的吸收; Picart等 (2017)对此模型进行数学分析和最优控制并证明溶 质浓度的存在性及唯一性。

Roose等在 Nye-Tinker-Barber模型基础上考虑 土壤颗粒表面的吸附和解析与浓度的关系, 通过尺
度变换、渐近展开、匹配的方法得到对流扩散方程 和扩散方程单根、根表面和根系统养分吸收通量的 近似解析解 (Roose et al., 2001; Roose \& Kirk, 2009)。随后Nowack等(2006)用3种地球化学计算工 具(ORCHESTRA、MIN3P和PHREEQC)检验解的合 理性。我们发现Roose的工作还有以下几个方面需要 改进和完善: 扩散方程求得的根表面养分通量的解 析解存在奇异性, 需要对时间进行平移; 没有给出 扩散方程和对流扩散方程根围区域浓度的解, 因此 我们不知道整个根围区域溶质浓度的变化; 没有将 对流扩散方程和扩散方程的通量解析解给出统一的 形式。本文在Roose工作的基础上试图解决以上问题, 并与数值模拟结果进行比较。

\section{Nye-Tinker-Barber模型}

20世纪70年代Nye、Tinker与Barber考虑土壤中 固态和液态养分的守恒, 提出的养分吸收模型 (Nye-Tinker-Barber模型) 是较为经典的单根养分吸 收模型(Nye \& Tinker, 1977; Barber, 1984)。

$$
\begin{aligned}
& \frac{\partial C_{\mathrm{S}}}{\partial t^{*}}=d_{\mathrm{S}} \\
& \frac{\partial}{\partial t^{*}}(\phi C)+\nabla \cdot(C U)=\nabla \cdot[\phi D \nabla C]-d_{\mathrm{S}}
\end{aligned}
$$

$C$ 是土壤中液态养分浓度, $C_{\mathrm{S}}$ 是土壤中固态养分浓 度, $\phi$ 是液体饱和度, $U$ 是水流达西通量, $D$ 是扩散 系数, $d_{\mathrm{S}}$ 是解析出被吸附离子的量。假设离子在土壤 颗粒表面的吸附和解吸与浓度成比例, 从而可以写 出吸附率、解吸率和固态、液态离子浓度的线性关 系表达式, 即:

$$
d_{\mathrm{S}}=k_{\mathrm{a}} C-k_{\mathrm{b}} C_{\mathrm{S}}
$$

$K_{\mathrm{a}}$ 是养分吸收速率, $K_{\mathrm{b}}$ 是养分离子解吸率。由于土壤 颗粒附着的离子解析时间较长, 因此,

$$
C_{\mathrm{S}}=b C
$$

其中: $b=K_{\mathrm{a}} / K_{\mathrm{b}}$ 为土壤缓冲率, 方程(1)和(2)可以 通过方程(3)和(4)变为:

$$
(\phi+\mathrm{b}) \frac{\partial C}{\partial t^{*}}+\nabla \cdot(C U)=\phi D \nabla^{2} C
$$

根表面的养分吸收遵循Michaelis-Menton动力学原理,

$$
\phi D \frac{\partial C}{\partial n}-\left(C U_{\mathrm{n}}\right)=\frac{F_{\mathrm{m}} C}{K_{\mathrm{m}}+C}, r^{*}=a
$$

在植物学中, $F_{\mathrm{m}}$ 和 $K_{\mathrm{m}}$ 分别是所考虑植物的生长速率 和酶促反应速度达最大值一半时的底物浓度。 $n$ 是根 表面指向土壤的单位矢量。 
达西通量为 $U=a V / r^{*}, V$ 是水进入根部的达 西通量, $r$ 是根半径, $a$ 是径向距离且满足 $\nabla \cdot U=0$ 。 因此在圆柱坐标里Nye-Tinker-Barber模型极坐标形 式为:

$$
\left\{\begin{array}{l}
(\phi+\mathrm{b}) \frac{\partial C}{\partial t^{*}}-\frac{a V}{r^{*}} \frac{\partial C}{\partial r^{*}}=\frac{\phi D}{r^{*}} \frac{\partial}{\partial r^{*}}\left(r * \frac{\partial C}{\partial r^{*}}\right) \\
\phi D \frac{\partial C}{\partial n}+V C=\frac{F_{\mathrm{m}} C}{K_{\mathrm{m}}+C}, r^{*}=a \\
C \rightarrow C_{0}, r^{*} \rightarrow \infty, t^{*} \rightarrow 0
\end{array}\right.
$$

引入量纲 $C=K_{\mathrm{m}} c, r^{*}=a r, t^{*}=(\phi+D) a^{2} t / \phi D$, 对 模型(5)进行无量纲化处理变为:

$$
\begin{aligned}
& \frac{\partial c}{\partial t}-P e \frac{1}{r} \frac{\partial c}{\partial r}=\frac{1}{r} \frac{\partial}{\partial r}\left(r \frac{\partial c}{\partial r}\right) \\
& \frac{\partial c}{\partial r}+P e \quad c=\frac{\lambda c}{1+c}, r=1 \\
& c=c_{\infty}, r=r_{\infty} \\
& c=c_{\infty}, t \rightarrow 0
\end{aligned}
$$

其中, 无量纲参数pecelet数(对流速率与扩散速率之 比) $P e=a V / \phi D$, 养分吸收通量系数 $\lambda=\frac{F_{\mathrm{m}} a}{\phi D K_{\mathrm{m}}}$, 无 量纲远场区域液态养分浓度和初始浓度 $c_{\infty}=C_{0} /$ $K_{\mathrm{m}}$ 。

由左边界条件(7)可知, 根表面的无量纲养分吸 收通量 $F_{\text {tot }}(t)$ 为:

$$
F_{\text {tot }}(t)=\left.\frac{\lambda c}{1+c}\right|_{r=1}
$$

\section{2 模型求解}

本文将整个根区分为远场区域和近场区域，在 远场区域对方程和右边界条件进行尺度变换, 并引 入相似变量进行求解。在近场区域对方程和左边界 条件进行尺度变换, 进行求解。对远场解和近场解 中的常数项及一次项进行匹配, 从而获得根表面的 养分吸收通量近似解析解和溶质浓度的全局近似解 析解。

\section{1 远场区域}

对模型中的时间变量 $t$ 和空间变量 $r$ 进行尺度变 换, 并引入相似变量 $\eta$ 。

$$
t=\frac{\tau}{\sigma^{2}}, r=\frac{R}{\sigma}, \eta=\frac{R^{2}}{\tau}
$$

$c(r, t)$ 可以写为关于 $\eta$ 的函数, $c(r, t)=c_{\infty} f(r, t)=$ $c_{\infty} f(\eta)$ 。

$$
\left\{\begin{array}{l}
\frac{\partial c}{\partial t}=-c_{\infty} f^{\prime}(\eta) \frac{r^{2}}{\tau^{2}} \\
\frac{\partial c}{\partial r}=-c_{\infty} f^{\prime}(\eta) \frac{2 r}{\tau} \\
\frac{\partial^{2} c}{\partial t^{2}}=\frac{4}{r} c_{\infty} f^{\prime}(\eta)+c_{\infty} f^{\prime \prime}(\eta) \frac{4 r^{2}}{\tau^{2}}
\end{array}\right.
$$

将方程(11)代入方程(6)中, 可以得到:

$$
-\eta f^{\prime}(\eta)=2(P e+2) f^{\prime}(\eta)+f^{\prime \prime}(\eta) \eta
$$

联立方程(12)和右边界条件(8)求解，可得远场区域 的养分浓度：

$$
c(r, t)=c_{\infty}-2^{-P e} B \text { Gamma }\left[-\frac{P e}{2}, \frac{r^{2}}{4 t}\right]
$$

在 $r=1$ 处把方程(13)渐近展开到一阶:

$$
\begin{aligned}
c(r, t)= & c_{\infty}-2^{-P e} B \text { Gamma }\left[-\frac{P e}{2}, \frac{1}{4 t}\right]+ \\
& 2 B e^{-\frac{1}{4 t}} \frac{P e}{2}(r-1)+O(r-1)^{2}
\end{aligned}
$$

\section{2 近场区域}

对模型中的时间尺度 $t$ 进行尺度变换:

$$
t=\frac{\tau}{\sigma^{2}}
$$

将方程(15)代入方程(6)得到:

$$
\sigma^{2} \frac{\partial c}{\partial \tau}=\frac{1}{r} \frac{\partial}{\partial r}\left(r \frac{\partial c}{\partial r}\right)+P e \frac{1}{r} \frac{\partial c}{\partial r}
$$

如果 $\sigma<<1$, 方程(16)可以简化为:

$$
0=\frac{1}{r} \frac{\partial}{\partial r}\left(r \frac{\partial c}{\partial r}\right)+P e \frac{1}{r} \frac{\partial c}{\partial r}
$$

通解为: $c(r, t)=c_{1}-\frac{c_{0}}{P e} r^{-P e}$ 其中 $c_{1}$ 和 $c_{0}$ 是关于 $t$ 的 函数, 根据模型的边界条件 $\left(F=\left.\frac{\partial c}{\partial r}\right|_{r=1}=c_{0}\right)$, 可得 $F=\left.\frac{c_{0}}{r}\right|_{r=1}=c_{0}$, 所以根表面附近的养分浓度为:

$$
c(r, t)=c_{1}-\frac{F}{P e} r^{-P e}
$$

在 $r=1$ 处把方程(18)渐近展开到二阶,

$$
c(r, t)=c_{1}-\frac{F}{P e}+(r-1) F+O(r-1)^{3}
$$

\section{3 解的匹配}

方程(14)和方程(19)是关于 $r=1$ 的级数形式, 匹配它们的常数项和一次项, 进行待定函数求解:

$$
\begin{aligned}
& c_{1}-\frac{F}{P e}=c_{\infty}-2^{-P e} B \text { Gamma }\left[-\frac{P e}{2}, \frac{1}{4 t}\right] \\
& F=2 B e^{-\frac{1}{4 t}} t^{\frac{P e}{2}}
\end{aligned}
$$




$$
\begin{aligned}
& F=\frac{2 c_{\infty}\left[\lambda-P e\left(1+c_{\infty}\right)\right]}{h\left(\lambda-P e c_{\infty}\right)+(1-P e h)\left(1+c_{\infty}\right)+\sqrt{\left(1+c_{\infty}\right)^{2}+h^{2}(\lambda-P e)^{2}-2 h\left[\lambda\left(-1+c_{\infty}\right)+P e\left(1+c_{\infty}\right)\right]}} \\
& F_{\text {tot }}=F+P e\left(c_{\infty}-h F\right)=\frac{2 \lambda c_{\infty}}{\left(1+c_{\infty}\right)+(\lambda-P e) h+\sqrt{\left(1+c_{\infty}\right)^{2}+h^{2}(\lambda-P e)^{2}-2 h\left[\lambda\left(-1+c_{\infty}\right)+P e\left(1+c_{\infty}\right)\right]}} \\
& c(r, t)=c_{\infty}+\frac{2^{-P e} c_{\infty} t^{-\frac{P e}{2}} e^{\frac{1}{4 t}}\left[P e\left(1+c_{\infty}\right)-\lambda\right] G a m m a[-P e / 2,1 / 4 t]}{h\left(\lambda-P e c_{\infty}\right)+(1-P e h)\left(1+c_{\infty}\right)+\sqrt{\left(1+c_{\infty}\right)^{2}+h^{2}(\lambda-P e)^{2}-2 h\left[\lambda\left(c_{\infty}-1\right)+P e\left(1+c_{\infty}\right)\right]}} \\
& F_{\text {tot }}(t)=\frac{2 \lambda c_{\infty}}{1+c_{\infty}-\lambda l+\sqrt{\left(1+c_{\infty}-\lambda l\right)^{2}+4 \lambda l c_{\infty}}} \\
& c(r, t)=c_{\infty}+\frac{\lambda c_{\infty} e^{\frac{1}{4 t}} \operatorname{ExpIntergralEi}[-1 / 4 t]}{-l \lambda+\left(1+c_{\infty}\right)+\sqrt{\left(1+c_{\infty}\right)^{2}+(\lambda l)^{2}+2 l \lambda\left(c_{\infty}-1\right)}}
\end{aligned}
$$

由边界条件(7)解得方程(20)。根据模型的边界 条件(7), 得到土壤中根表面的无量纲养分吸收通量 为方程(21)。由方程(14)得到土壤中根围区域无量纲 液态养分浓度的全局近似解析解为方程(22), 其中: $h=1 / 2^{1+P e} e^{\frac{1}{4 t}} t-\frac{P e}{2} G a m m a[-P e / 2,1 / 4 t]$ 。

通常情况下, 土壤里面的对流项很小, 一般认 为是 $P e=0$, 由方程(21)和(22)我们推出 $P e=0$ 时, 土壤中根表面的无量纲养分吸收通量 $F_{\mathrm{tot}}(t)$ 和液态 养分浓度的全局近似解析解 $c(r, t)$ 分别为方程(23)和 (24), 其中: $l=\frac{1}{2} e^{\frac{1}{4 t}}$ ExpIntergralEi $[-1 / 4 t]$ 。

Roose等在2001求解扩散方程的吸收通量的近 似解析解为:

$$
F_{\text {tot }}(t)=\frac{\lambda\left[c_{\infty}+F(\gamma-\ln 4 t) / 2\right]}{1+c_{\infty}+F(\gamma-\ln 4 t) / 2}
$$

当 $t=0$ 时 $F_{\text {tot }}=0$ 或 $\lambda$, 并不满足边界 $F_{\text {tot }}=\frac{\lambda c_{\infty}}{\left(1+c_{\infty}\right)}$, 需要时间的平移解决奇性, $\ln 4 t$ 应变成 $\ln \left(4 t+\mathrm{e}^{\gamma}\right)$ 。

在 $P e=0$ 的情况下, 我们使用方程(14)和(7), 得出

$$
F_{\text {tot }}=\frac{\lambda\left(c_{\infty}-e^{\frac{1}{4 t}} \text { ExpIntergralEi }\left[-\frac{1}{4 t}\right] F\right)}{1+c_{\infty}-e^{\frac{1}{4 t}} \text { ExpIntergralEi }\left[-\frac{1}{4 t}\right] F}
$$

由于: $\lim _{t \rightarrow 0} e^{\frac{1}{4 t}}$ ExpIntergralEi $[-1 / 4 t]=0$, 因此方程 (25)满足边界条件 $F_{\text {tot }}=\lambda c_{\infty} /\left(1+c_{\infty}\right)$, 且没有奇性。
方程(22)和(24)是对流扩散方程和扩散方程根 围区域溶质浓度的全局近似解析解, 方程(21)和(23) 是对流扩散方程和扩散方程根表面养分吸收通量的 近似解析解。由于Gamma $[0,1 / 4 t]=$-ExpIntergral$E i[1 / 4 t]$, 因此方程(21)、(22)、(23)、(24)具有统一 的形式。

\section{3 数值模拟}

在方程(6)中, 我们选取一般土壤特征参数值 (扩散系数 $D=0.3 \times 10^{-5} \mathrm{~cm}^{2} \cdot \mathrm{s}^{-1}$, 土壤含水量 $\phi=0.3$, 水分通量 $V=10^{-7} \mathrm{~cm}^{2} \cdot \mathrm{s}^{-1}$ ) 植物(玉米)相关的参数 (根 半径 $a=0.02 \mathrm{~cm}$, 根围 $r_{\text {max }}=3 \mathrm{~cm}$ )。我们对方程(6) 采取迎风差分格式进行数值模拟, 其中时间步长为 0.004 , 靠近根表面的空间步长为 0.4 , 远离根表面的 空间步长为 4 。模拟不同元素(钾、氮、硫、磷、镁、 钙)通量随时间变化情况和不同养分元素(钾和氮)浓 度在不同时间(1天、7天、13天、19天)的变化情况。

如图1所示, 在扩散方程中, 6种元素的通量呈 现两种衰减趋势, 其中钾、磷、镁通量在短时间内 迅速减少，氮、硫、钙的通量则随时间的变化而缓 慢下降。根表面养分通量的近似解析解方程(23) 和Roose等(2001)的解析解(2.24)接近, 但均高于数 值解。

如图2所示, 在对流扩散方程中, 镁的通量呈现 不同的变化趋势。在根表面Roose等(2001)的解析解 要比数值解大很多, 但本文养分通量的近似解析解 方程(21)较Roose等(2001)的近似解析解更接近于数 值解。 
如图3所示, 在扩散方程中, 在不同时间钾在根 表面的浓度随距离的变化被快速吸收。在近场区域, Roose 等(2001)的解析解(2.21)与本文的近似解析 解方程(24)接近但均高于数值解, 但随后与数值 解重合。

如图4所示, 在对流扩散方程中, 在近场区域,
本文的近似解析解方程(22)接近于数值解，随后与 数值解重合(Roose等(2001)没有给出对流扩散方程 根围的溶质浓度)。

在图5中，在扩散方程中，在同一时刻氮在根表 面的浓度随距离的变化而缓慢变化。在近场区域, Roose等(2001)的解析解(2.21)与本文的近似解析解
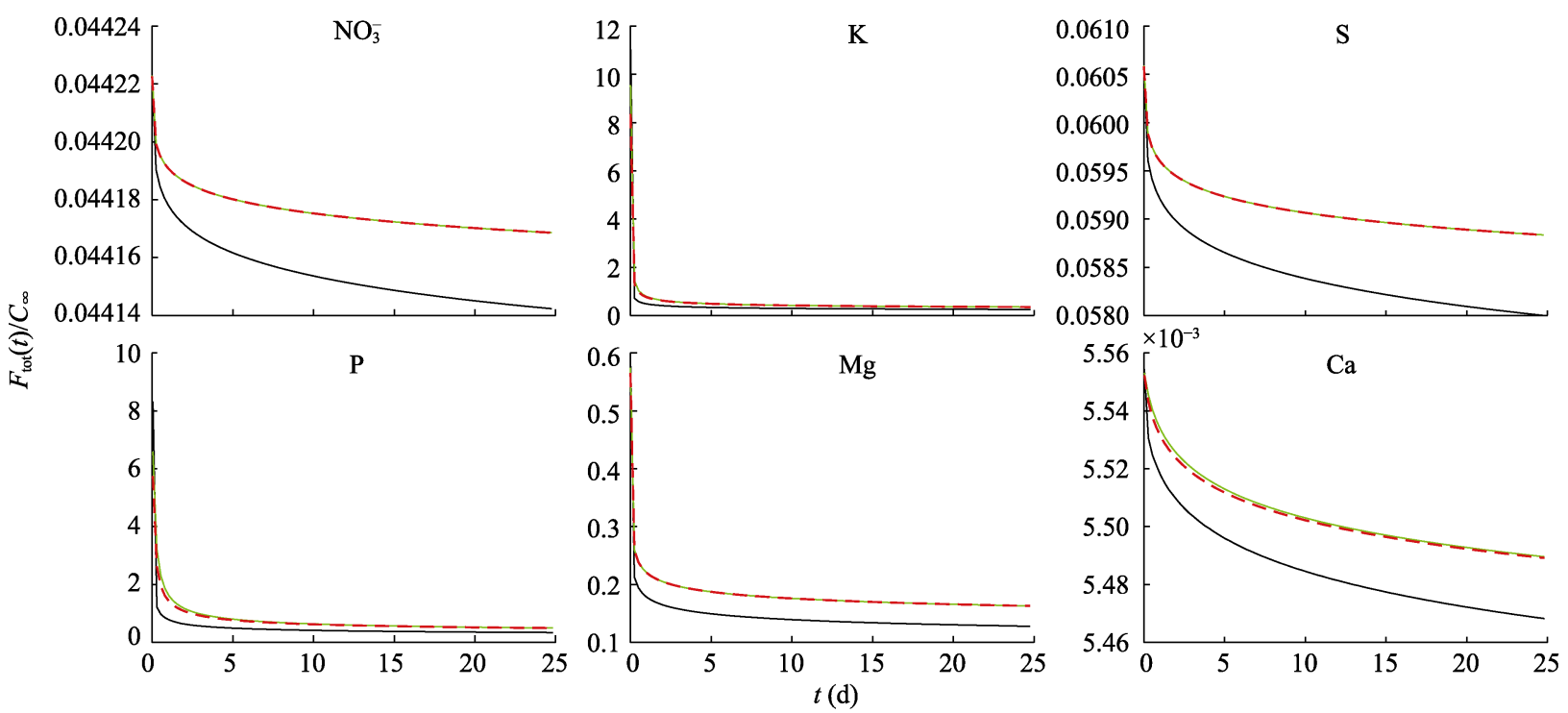

—数值通量 Numerical flux — Roose (2001)方程(2.24) Equation (2.24) of Roose (2001)

1) - - 本文方程(23) Equation (23) in this paper

图1 扩散方程中 6 种元素通量随时间的变化趋势。 $C_{\infty}$ 是初始浓度, 6种元素的初始浓度分别为5、0.046、0.1、0.0029、0.001、 $0.008 \mu \mathrm{mol} \cdot \mathrm{cm}^{-3} ; F_{\mathrm{tot}}(t)$ 是养分吸收通量; $t$ 是时间，以 25 天为例。

Fig. 1 Changes in the fluxes of six elements with time in diffusion Nye-Tinker-Barber model. $c_{\propto}$ is the initial concentration, with given values of $5,0.046,0.1,0.0029,0.001,0.008 \mu \mathrm{mol} \cdot \mathrm{cm}^{-3}$ for the six elements; $F_{\text {tot }}(t)$ is the element uptake flux; $t$ is time, with a duration of 25 days as an example.
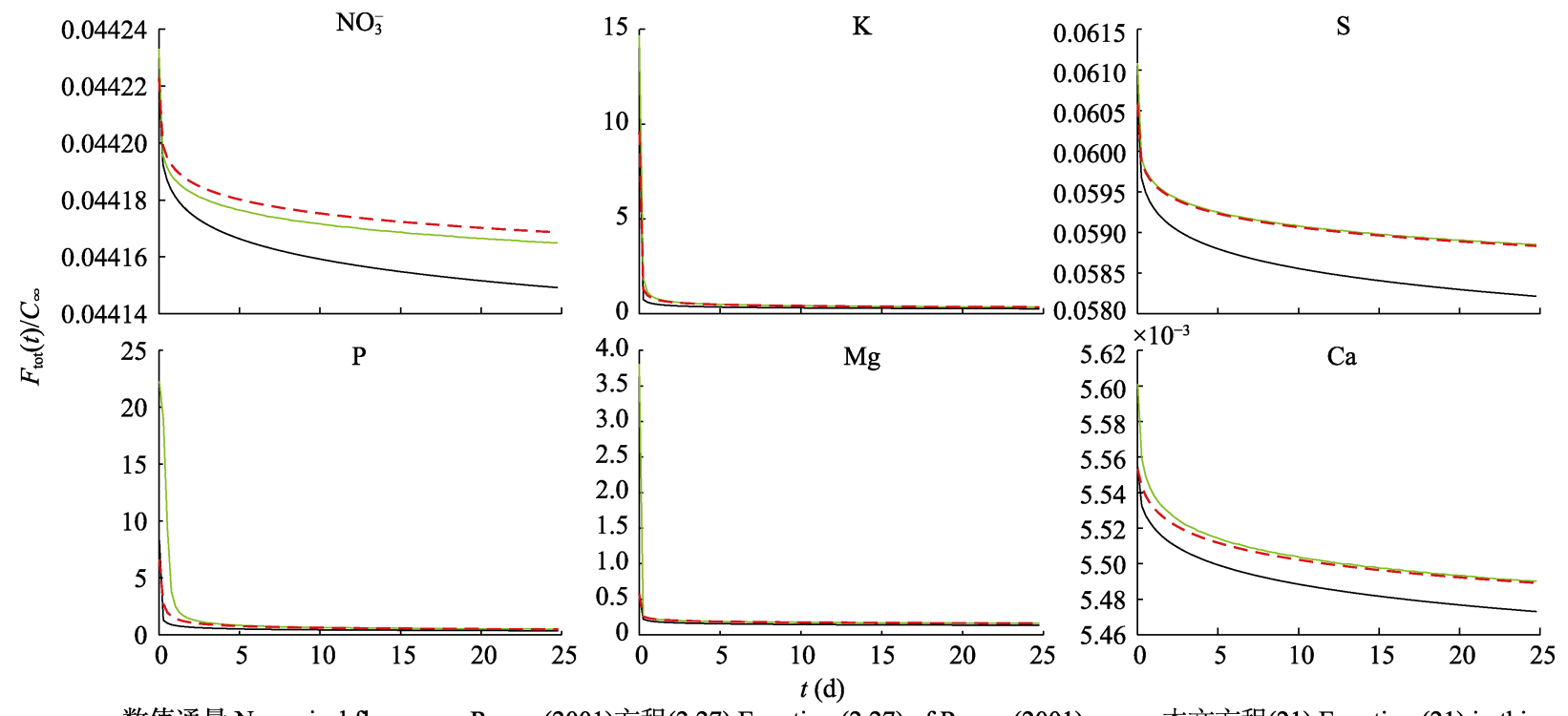

—数值通量 Numerical flux — Roose (2001)方程(2.27) Equation (2.27) of Roose (2001)

- - 本文方程(21) Equation (21) in this paper

图2 对流扩散方程中6种元素通量随时间的变化趋势。 $C_{\propto}$ 是初始浓度, 6种元素的初始浓度分别为5、0.046、0.1、0.0029、0.001、 $0.008 \mu \mathrm{mol} \cdot \mathrm{cm}^{-3} ; F_{\mathrm{tot}}(t)$ 是养分吸收通量; $t$ 是时间，以 25 天为例。

Fig. 2 Changes in the fluxes of six elements with time in convection-diffusion Nye-Tinker-Barber model. $c_{\propto}$ is the initial concentration, with given values of $5,0.046,0.1,0.0029,0.001,0.008 \mu \mathrm{mol} \cdot \mathrm{cm}^{-3}$ for the six elements; $F_{\text {tot }}(t)$ is the element uptake flux; $t$ is time, with a duration of 25 days as an example. 
方程(24)重合, 在根表面与数值解接近, 随后与数 值解重合。

在图6中，在对流扩散方程中，在近场区域，本

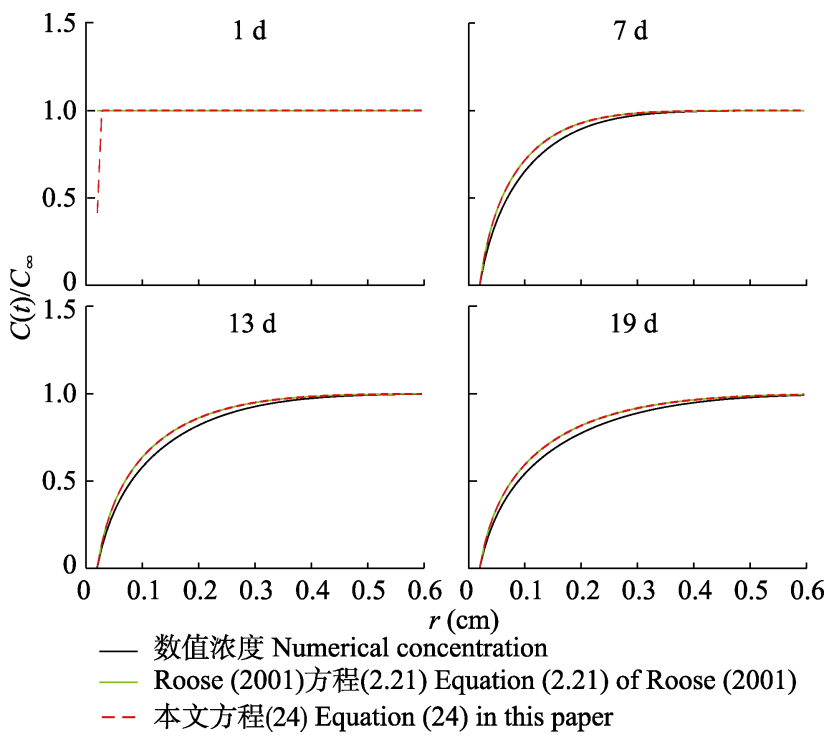

图3 扩散方程中 $1 、 7 、 13 、 19$ 天钾浓度随距离的变化趋势。 $c(t)$ 是养分浓度; $c_{\infty}$ 是养分初始浓度，钾的初始浓度为 0.046 $\mu \mathrm{mol} \cdot \mathrm{cm}^{-3} ; r$ 是到根表面的距离。

Fig. 3 Changes in potassium concentration with distance for 1, 7, 13 and 19 in diffusion Nye-Tinker- Barber model. $c(t)$ is the nutrient concentration; $c_{\infty}$ is the initial concentration, with a given value of $0.046 \mu \mathrm{mol} \cdot \mathrm{cm}^{-3}$ for potassium, $r$ is the distance to the root surface.

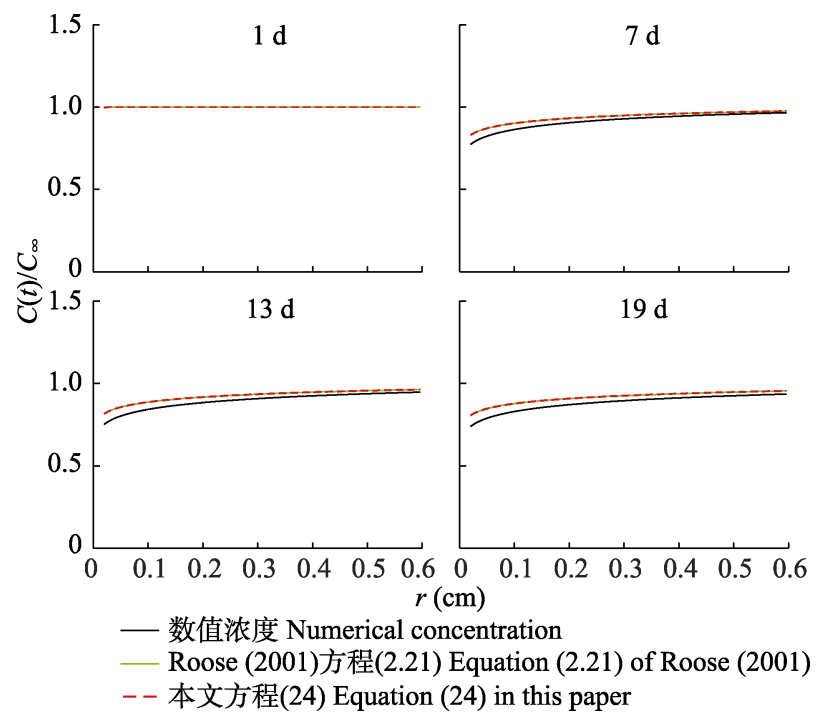

图5 扩散方程中 $1 、 7 、 13 、 19$ 天氮浓度随距离的变化趋势。 $c(t)$ 是养分浓度; $c_{\propto}$ 是养分初始浓度，氮的初始浓度为 5 $\mu \mathrm{mol} \cdot \mathrm{cm}^{-3} ; r$ 是根表面的距离。

Fig. 5 Changes in nitrogen concentration with distance for 1, 7,13 and $19 \mathrm{~d}$ in diffusion Nye-Tinker- Barber model. $c(t)$ is the nutrient concentration; $c_{\infty}$ is the initial concentration, with a given value of $5 \mu \mathrm{mol} \cdot \mathrm{cm}^{-3}$ for nitrogen; $r$ is the distance to the root surface.
文的近似解析解方程(22)接近于数值解, 随后与数 值解重合(Roose等(2001)没有给出对流扩散方程根 围的溶质浓度)。

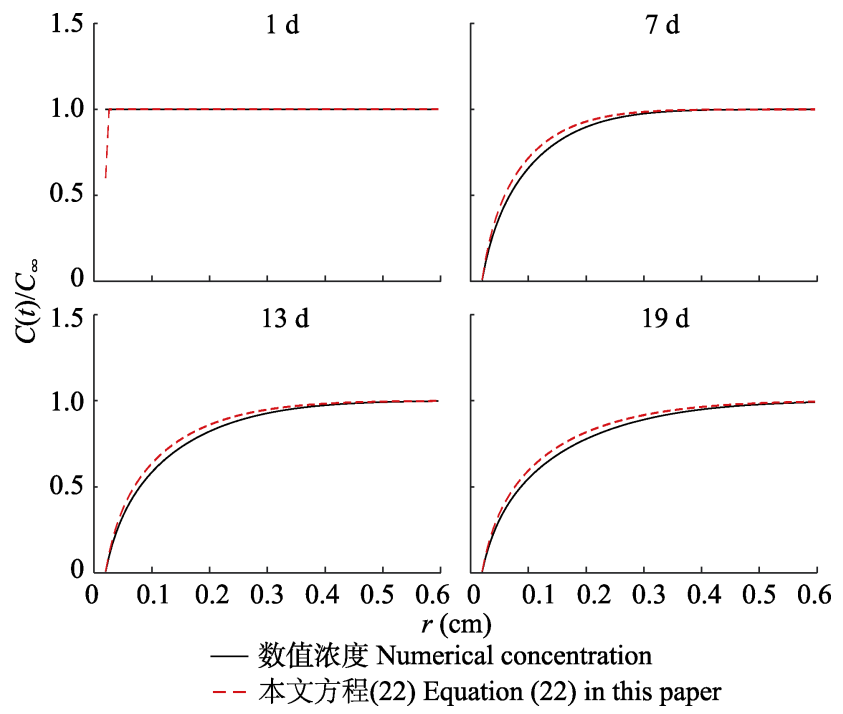

图4 对流扩散方程中 $1 、 7 、 13 、 19$ 天钾液态浓度随距离的变 化趋势。 $c(t)$ 是养分浓度; $c_{\infty}$ 是养分初始浓度, 钾的初始浓度为 $0.046 \mu \mathrm{mol} \cdot \mathrm{cm}^{-3} ; r$ 是到根表面的距离。

Fig. 4 Changes in potassium concentration with distance for 1, 7, 13 and 19 in convection-diffusion Nye-Tinker- Barber model. $c(t)$ is the nutrient concentration; $c_{\propto c}$ is the initial concentration, with a given value of $0.046 \mu \mathrm{mol} \cdot \mathrm{cm}^{-3}$ for potassium; $r$ is the distance to the root surface.

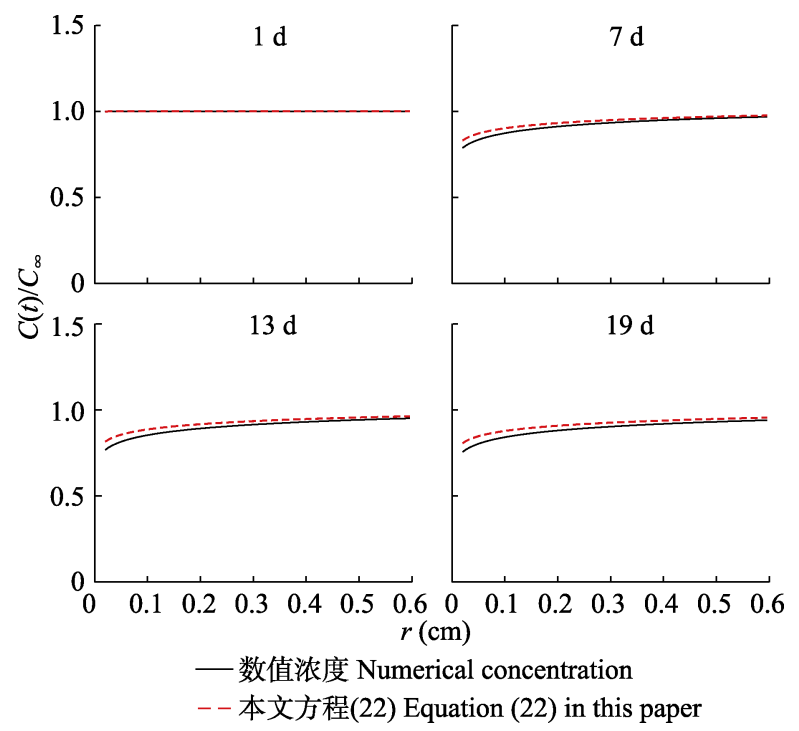

图6 对流扩散方程中 $1 、 7 、 13 、 19$ 天氮液态浓度随距离的变 化趋势。 $c(t)$ 是养分浓度; $c_{\infty}$ 是养分初始浓度, 氮的初始浓度为 $5 \mu \mathrm{mol} \cdot \mathrm{cm}^{-3} ; r$ 是根表面的距离。

Fig. 6 Changes in nitrogen concentration with distance for 1, 7, 13 and $19 \mathrm{~d}$ in convection-diffusion Nye- Tinker-Barber model. $c(t)$ is the nutrient concentration; $c_{\propto}$ is the initial concentration, with a given value of $5 \mu \mathrm{mol} \cdot \mathrm{cm}^{-3} ; r$ is the distance to the root surface. 


\section{4 结论}

本文用Nye-Tinker-Barber模型来求解植物根系 表面的养分吸收通量和根围溶质浓度的近似解析 解。通过把远场解在根表面展开并与近场解匹配, 不仅获得根表面的养分吸收通量近似解析解, 还求 解出根际周围溶质浓度的全局近似解析解, 从而解 决了Roose解中的奇性问题, 并且本文给出了对流 扩散方程和扩散方程根表面养分通量和溶质浓度全 局近似解析解的统一形式。

通过数值模拟对比, 在扩散模型和对流扩散模 型中, 我们都发现6种元素的通量随时间的增加而 衰减, 且呈现两种变化趋势, 其中钾、磷、镁通量在 短时间内迅速减少, 镁通量在扩散方程和对流扩散 方程呈现不同变化趋势, 氮、硫、钙的通量则随时 间的变化而缓慢下降, 在对流扩散方程中本文的解 析解较Roose的解析解更接近于数值解; 近场区域 对流扩散模型和扩散模型中, 两种元素浓度全局解 析解的变化趋势与数值解一致, 先高于数值解, 随 后与数值解重合。

致谢 感谢福建师范大学创新团队项目 (19120/ Z1607219016和IRTL1206)和分析数学及应用重点 实验室项目(ZGD1707233和ZGD1707222711)资助。 感谢穆怡教授、沈建和教授和陈建清教授的指导和 帮助。

\section{参考文献}

Barber SA (1984). Soil nutrient bioavailability: A mechanistic approach. Quarterly Review of Biology, 161, 140-141.

Badge R, Adlakha N (2017). Two-dimensional finite element model to study the effect of water flux on the nitrate dynamics in the rhizosphere of a maize root. International Journal of Biomathematics, 10, 333-351.

Bouldin DR (1961). Mathematical description of diffusion processes in the soil-plant system. Soil Science Society of America Journal, 25, 476-480.

Chen H, Feng Y, Zhou JM, Xu ZG, Lian C, Guo QR (2013). Research advance of plant root biology. World Forestry Research, 26(5), 25-29. [陈红, 冯云, 周建梅, 徐振国, 廉超, 郭起荣 (2013). 植物根系生物学研究进展. 世界 林业研究, 26(5), 25-29.]

Epstein E (1972). Mineral Nutrition of Plants: Principles and Perspectives. John Wiley \& Sons, New York.
Kumar A, Hafiz A (2011). A mathematical description of pollutant uptake in plants by single cylindrical root. American Journal of Experimental Agriculture, 1, 265-280.

Meng HS, Feng JY, Hu DD, Qin WM, Yan L (2017). Root system development and nutrient absorption in plant. Journal of Shanxi Agricultural Sciences, 45, 1048-1052. [蒙 好生, 冯娇银, 胡冬冬, 秦武明, 严理 (2017). 植物根 系发育与养分的吸收. 山西农业科学, 45, 1048- 1052.]

Nowack B, Mayer KU, Oswald SE, van Beinum W, Appelo CAJ, Jacques D, Seuntjens P, Gérard F, Jaillard B, Schnepf A, Roose T (2006). Verification and intercomparison of reactive transport codes to describe root-uptake. Plant and soil, 285, 305-321.

Nye PH, Spiers JA (1964). Simultaneous diffusion and mass flow to plant roots. In: Proceedings of the 8th International Congress of Soil Science. Rompresfilatelia, Bucharest, Hungary. 535-544.

Nye PH, Tinker PB (1977). Solute Movement in the Soil-Root System. Blackwell Scientific Publications, Oxford, UK.

Olsen SR, Kemper WD, Jackson RD (1962). Phosphate diffusion to plant roots. Soil Science Society of America Journal, 26, 222-227.

Picart D, Ozier-Lafontaine H, Omrane A, Louison L (2017). Modeling plant nutrient uptake: Mathematical analysis and optimal control. Evolution Equations and Control Theory, 4, 193-203.

Roose T, Fowler AC (2004). A mathematical model for water and nutrient uptake by plant root systems. Journal of Theoretical Biology, 228, 173-184.

Roose T, Fowler AC, Darrah PR (2001). A mathematical model of plant nutrient uptake. Journal of Mathematical Biology, 42, 347-360.

Roose T, Kirk DJD (2009). The solution of convection-diffusion equations for solute transport to plant roots. Plant and Soil, 316, 257-264.

Schnepf A, Jones D, Roose T (2011). Modelling nutrient uptake by individual hyphae of arbuscular mycorrhizal fungi: Temporal and spatial scales for an experimental design. Bulletin of Mathematical Biology, 73, 2175-2200.

Schnepf A, Roose T (2006). Modeling the contribution of arbuscular mycorrhizal fungi to plant phosphate uptake. New Phytologist, 171, 669-682.

Zygalakis KC, Kirk GJ, Jones DL, Wissuwa M, Roose T (2011). A dual porosity model of nutrient uptake by root hairs. New Phytologist, 192, 676-688.

Zygalakis KC, Roose T (2012). A mathematical model for investigating the effect of cluster roots on plant nutrient uptake. The European Physical Journal Special Topics, 204, 103-118.

责任编委: 周广胜 责任编辑: 李 敏 